Revue bibliographique pour le domaine irano-aryen

\title{
Matteo De Chiara, Enrico G. Raffaelli (a cura di). Philippe Gignoux. Mazdéens et chrétiens en terre d'Iran à l'époque sassanide
}

\section{Christelle Jullien}

\author{
(2) OpenEdition \\ Journals \\ Édition électronique \\ URL : http://journals.openedition.org/abstractairanica/43403 \\ DOI : $10.4000 /$ abstractairanica.43403 \\ ISBN : 1961-960X \\ ISSN : 1961-960X \\ Éditeur : \\ CNRS (UMR 7528 Mondes iraniens et indiens), Éditions de l'IFRI
}

\section{Référence électronique}

Christelle Jullien, « Matteo De Chiara, Enrico G. Raffaelli (a cura di). Philippe Gignoux. Mazdéens et chrétiens en terre d'Iran à l'époque sassanide », Abstracta Iranica [En ligne], Volume 37-38-39 | 2018, document 2, mis en ligne le 30 décembre 2018, consulté le 01 octobre 2020. URL : http://

journals.openedition.org/abstractairanica/43403; DOI : https://doi.org/10.4000/abstractairanica. 43403

Ce document a été généré automatiquement le 1 octobre 2020.

Tous droits réservés 


\title{
Matteo De Chiara, Enrico G. Raffaelli (a cura di). Philippe Gignoux. Mazdéens et chrétiens en terre d'Iran à l'époque sassanide
}

\author{
Christelle Jullien
}

\section{RÉFÉRENCE}

Philippe Gignoux. Mazdéens et chrétiens en terre d'Iran à l'époque sassanide. Matteo De Chiara, Enrico G. Raffaelli (a cura di), (Serie Orientale Roma, N. S. 3), Roma : Scienze e Lettere, 2014, 682 p., ill., ISBN 978-88-6687-066-1.

1 Ce volume honore la longue et productive carrière scientifique de Philippe Gignoux en réunissant 42 contributions qu'il réalisa entre 1974 et 2005 sur les sources primaires sassanides et sur les "religions de l'Iran ancien", plus spécifiquement les communautés zoroastriennes et chrétiennes dans l'empire sassanide. Il est préfacé par Marco Mancini et Adriano V. Rossi ; un avant-propos signé par Philippe Gignoux permet de présenter l'ensemble des études choisies. Les éditeurs les ont classées selon trois rubriques thématiques :

2 La première comprend quatorze articles disposés en ordre chronologique ayant essentiellement trait à des questions d'épigraphie moyen-perse sur des sceaux et des bulles, et sur des inscriptions sassanides («Notes d'épigraphie et d'histoire sassanides ", Mélanges linguistiques offerts à Émile Benveniste, Paris, 1975, p. 213-223; en coll. avec L. Kalus, «Les formules des sceaux sassanides et islamiques: Continuité ou mutation?», Studia Iranica 11, 1982, p. 123-153 ; «Les bulles sassanides de qasr-i Abu Nasr (collection du Musée de Téhéran)», Mémorial Jean de Menasce, Louvain, 1974, p. 169-187 ; «Les bulles sasanides de qasr-i Abu Nasr (collection du Metropolitan Museum of 
Art) », Papers in Honour of Professor Mary Boyce, [Acta Iranica 24], Liège, 1985, p. 195-215 ; en coll. avec R. Gyselen, "Sceaux de femmes à l'époque sassanide », Archaeologia Iranica et Orientalis. Miscellanea in honorem Louis Vanden Berghe, Gent, 1989, II, p. 877-896; « D'Abnūn à Māhān : étude de deux inscriptions sassanides », Studia Iranica 20, 1991, p. 9-22 ; « La signification du bas-relief sassanide de Sar-Mašhad », dans W. Skalmowski, A. van Tongerloo (eds), Medioiranica. Proceedings of the International Colloquium organized by the Katholieke Universiteit Leuven from the 21st to the 23rd of May 1990, [Orientalia Lovaniensia Analecta 48], Leuven, 1993, p. 71-78 ; "Les inscriptions en moyen-perse de Bandīān », Studia Iranica 27, 1998, p. 251-258 ; « On the New Pahlavi Documents from Central Asia », Indologica Taurinensia 23-24, 1997-1998 [2000]) [= Professor Gregory M. Bongard-Levin Felicitation Volume], p. 543-549). Quelques articles ont trait à des questions d'onomastique et de prosopographie ("Éléments de prosopographie de quelques Mōbads sasanides", Journal Asiatique 270, 1982, p. 257-269; "Éléments de prosopographie: II. Les possesseurs de coupes sasanides », Studia Iranica 13, 1984, p. 19-40

; "Noms d'utensiles (argenterie et poterie) en moyen-iranien », dans Akira Haneda (éd.), Documents et archives provenant de l'Asie centrale. Actes du Colloque franco-japonais Kyoto 4-8 octobre $1988 \quad, \quad$ Kyoto, $\quad 1990, \quad$ p. 71-86 ), et plus accessoirement de toponymie (" Nouveaux toponymes sassanides ", Journal Asiatique 262, 1974, p. 299-304). Y a été adjointe une étude de numismatique («Les nouvelles monnaies de Shāpūr II ", Studia Iranica 19, 1990, p. 195-204 ).

3 Suit une seconde partie comportant dix-sept études rassemblées sous le thème d' « Histoire du mazdéisme et religions comparées » : « La signification du voyage extraterrestre dans l'eschatologie mazdéenne ", Mélanges d'histoire des religions offerts à HenriCharles Puech, Paris, 1974, 6. 63-69 ; “'Corps osseux et âme osseuse' : Essai sur le chamanisme dans l'Iran ancien», Journal
Asiatique
267,
1979 ,
p.
41-79

; «Les voyages chamaniques dans le monde iranien ", Monumentum Georg Morgenstierne, [Acta Iranica 21], Liège , 1981, p. 244-265 ; «Une ordalie par les lances en Iran », Revue de l'histoire des religions 200, 1983, p. $155-161$

; «Un témoin du syncrétisme mazdéen tardif: Le traité pehlevi des 'Sélections de Zādsparam' ", Transition Periods in Iranian History, [Studia Iranica. Cahier 5], Paris, 1987, p. 59-72

; " Dietary Laws in pre-Islamic and post-Sasanian Iran ", Jerusalem Studies in Arabic and Islam 17, 1994, p. 16-42; "La controverse dans le mazdéisme tardif », dans A. Le Boulluec (éd.), La controverse et ses formes, Paris, 1994, p. 127-149 ; « La doctrine du macrocosme-microcosme et ses origines gréco-gnostiques ", dans Petr Vavroušek (ed.), Iranian and Indo-European Studies, Memorial Volume of Otakar Klíma, Praha, 1994, p. 27-52 ; "The Notion of Soul (ruwān) in the Sasanian Mazdaeism ", K.R. Cama Oriental Institute, International Congress, Bombay, 1996, p. 23-35; «Quelques réflexions sur la représentation du paradis ", dans J.-P. Mahé, R. W. Thomson (eds), From Byzantium to Iran: Armenian Studies in Honour of Nina G. Garsoïan, Atlanta, 1996, p. 3-15 ; «L'identité zoroastrienne et le problème de la conversion », dans J.-Ch. Attias (éd.), De la conversion, Paris, 1997, p. 13-36 ; « On the Notion of Good Measure (paymān) and Other Related Philosophical Concepts from the Dēnkard III », K.R. Cama Oriental Institute, Third 
International Congress, Bombay, 2001, p. 278-286 ; « La continuité de la conduite morale dans le zoroastrisme », Revue de la Société Ernest Renan, Paris, 2000-2001, p. 57-69 ; « Les bases de la philosophie mazdéenne », Comptes Rendus de l'Académie des Inscriptions et Belles-Lettres, Paris, 2001, p. 117-129;

« Zamān ou le temps philosophique dans le Dēnkard III », dans C. G. Cereti, M. Maggi, E. Provasi (eds), Religious Themes and Texts of pre-Islamic Iran and Central Asia, Studies in Honour of Professor Gherardo Gnoli, [Beiträge zur Iranistik 24], Wiesbaden, 2003, p. 115-122 ; «L'eau et le feu dans le zoroastrisme », dans G. Capdeville (éd.), L'eau et le feu dans les religions antiques, Paris, 2004, p. 269-282; "Ancient Iranian Religions and Shamanism », dans M. N. Walter, E. J. N. Fridman (eds), Shamanism. An Encyclopedia of World Beliefs, Practices, and Culture, Santa Barbara, 2004, II, p. 529-532.

4 Une troisième rubrique comprend enfin onze réflexions relatives au christianisme syriaque, notamment en milieu sassanide: «Sur quelques noms propres iraniens transcrits en syriaque ", Mélanges offerts au R. P. François Graffin, S.J., Parole de l'Orient 6-7, 1975-1976,

p.

515-523

; «Titres et fonctions religieuses sasanides d'après les sources syriaques hagiographiques ", Acta Antiqua Academiae Scientiarum Hungaricae 28, Budapest, 1980 [1983], p. 191-203; «Les noms des signes du zodiaque en syriaque et leurs correspondants en moyen-perse et en mandéen ", Mélanges Antoine Guillaumont, [Cahiers d'Orientalisme XX], Genève, 1988, p. 299-304; "Imago Dei : De la théologie nestorienne à Ibn Al 'Arabī », dans Ph. Gignoux (éd.), Recurrent Patterns in Iranian Religions. From Mazdaism to Sufism, Proceedings of the Round Table Held in Bamberg (30th September-4th October 1991), [Studia Iranica. Cahier 11], Paris, 1992,

p.

13-27;

«Le traité syriaque anonyme sur les médications », dans R. Lavenant (éd.), Symposium Syriacum VII, [Orientalia Christiana Analecta 256], Roma, 1998, p. 725-733; " Anatomie et physiologie humaine chez un auteur syriaque, Ahūhdemmeh », Comptes Rendus de l'Académie des Inscriptions et Belles-Lettres, 1998, p. 231-242 ; «Sur quelques relations entre chrétiens et mazdéens d'après des sources syriaques ", $\begin{array}{lllll}\text { Studia Iranica } & 28, & \text { p. } & \text { 83-94 }\end{array}$ ; « Une typologie des miracles des saints et martyrs perses dans l'Iran sassanide », dans D. Aigle (sous la dir.), Miracle et Karāma, [Bibliothèque de l'école des Hautes études 109. Hagiographies médiévales comparées 2], Turnhout, 2000, p. 499-523; "Une croix de procession de Hérat inscrite en pehlevi ", Le Muséon 114, 2001, p. 291-304; "L'apport scientifique des chrétiens syriaques à l'Iran sassanide », Journal Asiatique 289, 2001 , p.

217-236 ; «La transmission de l'héritage grec aux Arabes par les Syriaques », Les Syriaques transmetteurs de civilisations, Colloque IX du C.E.R.O., Damas, 2004-2005, p. 53-65.

Des indices des principaux termes employés et des textes, ainsi qu'une bibliographie avec une liste d'abréviations sont donnés à la fin de cet ouvrage. La communauté scientifique est reconnaissante aux éditeurs de cette publication qui permet de réunir un bel ensemble d'études ayant largement contribué à faire avancer nos connaissances sur le milieu sassanide et chrétien oriental. Il constitue un ensemble de références rendues désormais aisément consultables. 


\section{AUTEURS}

\section{CHRISTELLE JULLIEN}

CNRS, Mondes iranien et indien, Paris 